NASA Technical Memorandum 102025

\title{
Optical Detectors for GaAs MMIC Integration: Technology Assessment
}

\author{
(AASA-TM-102025) CPTICAL CEYICICES FOR GaAS \\ $189-22020$ \\ WEIC INTEGEATICA: TECEACLCGI LSCESSEENT \\ (NaSA. Ievis besearch CenteI) 10 fCSCL OSA \\ $\begin{array}{ll}\text { Gnclas } & \text { Onc33 } \\ 0204401\end{array}$
}

P.C. Claspy and K.B. Bhasin

Lewis Research Center

Cleveland, Ohio

Prepared for the

1989 Technical Symposium on Aerospace Sensing

sponsored by the Society of Photo-Optical Instrumentation Engineers

Orlando, Florida, March 27-31, 1989 


\title{
OPTICAL DETECTORS FOR GaAs MMIC INTEGRATION: TECHNOLOGY ASSESSMENT
}

\author{
P. C. Claspy* and K. B. Bhasin \\ National Aeronautics and Space Administration \\ Lewis Research Center \\ Cleveland, Ohio 44135
}

\begin{abstract}
Fiber optic links are being considered to transmit digital and analog signals in phased array antenna feed networks in space communications systems. The radiating elements in these arrays will be GaAs monolithic microwave integrated circuits (MMIC's) in numbers ranging from a few hundred to several thousand. If such optical interconnects are to be practical it appears essential that the associated components, including detectors, be monolithically integrated on the same chip as the microwave circuitry. In this paper the general issue of monolithic integration of microwave and optoelectronic components is addressed from the point of view of fabrication technology and compatibility. Particular attention is given to the fabrication technology of various types of $\mathrm{GaAs}$ optical detectors that are designed to operate at a wavelength of $830 \mathrm{~nm}$.
\end{abstract}

\section{INTRODUCTION}

For many years attention has been given to a wide variety of system applications of optical fiber-based communications. These applications include telecommunications, internal and network-level computer interconnects, and microwave system interconnects. ${ }^{1,2}$ In each case there are advantages, ranging from speed improvements to reduction in cost and in space and power consumption, to be gained by appropriate monolithic integration of optical, optoelectronic, and electronic components. In an operational sense, however, perhaps the dominant factor in determining practical limits to the level of integration is compatibility of materials and processing technologies of the various components. This paper addresses the monolithic integration issue for the case of internal optical interconnection in microwave systems, with particular emphasis on optical detectors.

An underlying assumption in our discussion is that the system being considered here is, and will continue to be, basically a microwave system and that therefore if a choice is necessary, the dominant technology will be that which optimizes the performance of monolithic microwave integrated circuits (MMIC's), not that which optimizes the performance of the detectors. We have also assumed for purposes of this discussion that because of the short distances involved, optical interconnects in microwave systems will operate at a wavelength of $830 \mathrm{~nm}$. In the sections to follow we will consider microwave system requirements that may generate a need for optical interconnects, briefly review current MMIC fabrication technology, and examine the various types of detectors and their fabrication technologies. Finally, we will consider some recent examples of optoelectronic integration of a type that may be appropriate to microwave systems.

\section{MICROWAVE SYSTEM REOUIREMENTS}

As microwave systems for spacecraft and aircraft become more compact their limiting size can be increasingly dominated by the hardware, e.g., coaxial cables and waveguides, required for transmitting signal and control information between circuit boards and between individual subsystems. As an example of this problem consider the output section of a communications system that includes a phased array antenna, as shown schematically in Fig. 1. Although the details of layout and the location of specific functions are a subject of discussion among system designers, in such a system the microwave carrier

${ }^{*}$ National Research Council-NASA Senior Research Associate. 
must be modulated by the signal information that is to be transmitted, and the modulated carrier must must be delivered to each radiating element of the array with the correct relative phase and amplitude. Digitally controlled microwave phase shifter and variable gain amplifier MMIC's have been reported. ${ }^{3}$ As the figure suggests, both the signal information and the phase shifter and amplifier control signals as well as, perhaps, the modulated, phase shifted, amplified carrier are candidates for transmission by optical means. The former two are potentially wide bandwidth baseband signals while the latter is a narrow fractional bandwidth microwave frequency signal, so both the bandwidth and the operating frequency capabilities of an optical subsystem will be important.

Some considerations that must be made and which will affect the scale of integration of the MMIC's and the choice to use optical interconnects are:

- In a system such as that exemplified in Fig. 1, the microwave portions of the circuitry will be analog while the control portions will be digital. Since FETs for digital control and for analog microwave circuits are different, compromises may be necessary if integration is to be practical, independent of the optical interconnect issue.

- The scale of integration will depend upon material and processing compatibility, upon yield projections, and upon the system designer's requirements.

- If optical interconnects are to be used, detectors would act as the interface between the optical and the electrical subsystems.

- The overall system is primarily microwave, so the optical detectors and their associated circuitry must be material- and process-compatible with MMIC fabrication technology.

\section{CURRENT MMIC FABRICATION TECHNOLOGY}

To enable us to address the material and process compatibility issue let us first examine the current and probable future MMIC technology, as shown schematically in Fig. 2. At present perhaps the most commonly used technology is ion-implanted MESFET, directly on semi-insulating GaAs substrates, as shown in Fig. 2 (a). ${ }^{4}$ This process is well-developed, is economical to use, and gives reasonable yields, but it is limited in speed and noise characteristics by the physical properties of the wafer on which it is based. A second and somewhat similar technology bases the devices on a high purity GaAs layer that is grown epitaxially on a semi-insulating substrate, as shown in Fig. 2 (b). 5 The conducting layers may be produced by ion implantation into the grown layer or by use of a second, doped, epitaxial layer grown on the high purity layer. This material has higher device speed capabilities than the direct wafer implant, but its speed is still limited by scattering from the dopant impurities in the conducting layer. In both of these cases the device of choice for low noise, high power linear microwave circuits is the high-pinchoff-voltage D-MESFET, which is normally on, while for low power digital circuits the E-MESFET, which is normally off, or a low pinchoff voltage D-MESFET is typically chosen. ${ }^{6}$ Similar, but far from identical processes are used to produce the two devices, so the usual choice is an intermediate pinchoff DMESFET.

The structures shown in Figs. 2 (c) and (d) have been designed to overcome the scattering (by doping impurities) that limits device speed, and their use in MMIC's and high speed digital IC's is a topic of current research.7,8 In each material the conducting electrons originate from the n-type impurities in the $\mathrm{n}$-AlGaAs layer, but because of the band structure of the layered material conduction occurs in the undoped GaAs layer as a two-dimensional electron gas (2 DEG), resulting in higher electron mobilities and saturation velocities than can be obtained in doped material. It has been suggested that with a minor 
change in the heterostructure the same material can be used for both digital (E-MODFET) and analog devices (D-MODFET), with only slight differences in processing. ${ }^{7}$

\section{DETECTOR TYPES AND FABRICATION TECHNOLOGIES}

Planar, two-terminal optical detectors that are appropriate for consideration in MMIC interconnects can be classified into three types, namely back-to-back diodes, single diodes, and photoconductors, as shown schematically in Fig. 3. Fig. 3 (a) shows a MSM (metal-semiconductor-metal) detector as a representative of the back-to-back diode. Both contacts of the MSM device are Schottky-type blocking contacts, with the barrier height, and therefore the dark current, being determined by the choice of contact metal. The MSM detector can be fabricated directly on a semi insulating wafer or on a high purity epitaxial layer, with no ion implantation requirement. Fig. 3 (b) represents a p-i-n (p-type, intrinsic, n-type) photodiode that must be produced by ion implantation. The contacts on this device are ohmic to the p-type and $n$-type materials. The MSM and $\mathrm{p}-\mathrm{i}-\mathrm{n}$ detectors have comparable response speeds and noise characteristics, and each produces one electron in an external circuit for each photon absorbed. The third detector type is the photoconductive detector, as shown schematically in Fig. 3 (c). This detector, like the MSM device, can be produced by liftoff and etching, although those produced on high purity material layers may be ion implanted. Both contacts are ohmic, non-blocking contacts and the device exhibits gain because of the combination of injecting contacts and different transit times for electrons and holes. In general photoconductive detectors have a short rise time, but the fall time in their response to a short pulse is long, with the result that their $3 \mathrm{~dB}$ frequency is lower than that of the other two types.

The operating characteristics of several representative and easily integrated detectors of each type and material, as gathered from the literature, are given in Table I.9-15 Since the operating wavelength has a significant effect on detector speed, a particular effort was made to select devices for which measurements have been made at the proposed operating wavelength of $830 \mathrm{~nm}$ even though faster GaAs-based detectors have been reported in the literature. The effect of wavelength on speed is shown schematically, for two different wavelengths, in Fig. 4. In each case the absorbing material is $\mathrm{GaAs}$, which has a band gap of $1.42 \mathrm{eV}$. The $\mathrm{e}^{-1}$ penetration depth of $830 \mathrm{~nm}$ light is $\approx 1 \mu \mathrm{m}$ while at $600 \mathrm{~nm}$ it is $\approx 0.2 \mu \mathrm{m}$. Thus at the longer wavelength significant numbers of photoelectrons are generated throughout the bulk of the detector, and they experience a depth-dependent electric field. At the shorter wavelength most of the photoelectrons are generated at the surface, where they experience a higher and relatively uniform electric field. The result is that a detector that has a bandwidth of, say, $100 \mathrm{GHz}$ at $600 \mathrm{~nm}$ may have only a $25 \mathrm{GHz}$ bandwidth at $830 \mathrm{~nm}$.

The table shows that response speeds up to about $7 \mathrm{GHz}$ are reasonably obtainable and that at least $20 \mathrm{GHz}$ can be achieved with MESFET technology. The only report, by the authors of this paper, describing the response at $830 \mathrm{~nm}$ of detectors fabricated on HEMT material like that used for microwave devices is for photoconductive detectors. Those detectors exhibited approximately a $2 \mathrm{GHz}$ bandwidth, and there was no indication of conduction by a 2 DEG. It may, however, be possible to increase the response speed of HEMT detectors - at the expense of responsivity - by specially tailoring the vertical profile of the material.

\section{CURRENT STATUS OF $830 \mathrm{NM}$ OPTOELECTRONIC INTEGRATION}

As indicated previously, there has been much discussion of the potential use of optical interconnects to MMIC's, where an optical receiver would be directly integrated on the same chip with, for example, microwave amplifiers or similar circuitry. The authors are unaware of any reports of such integrated circuits in the literature, but there have been reports describing monolithic MESFET optoelectronic integrated circuits (OEIC's) that have various capabilities for detecting and processing optically transmitted information and control signals. There have also been reports of the monolithic integration of (digital) control circuitry with microwave devices. Since such chips are the precursors of fully integrated OEIC- 
MMIC's, a set of appropriate examples, with varying degrees of complexity, of ion-implanted and epitaxial OEIC's have been selected from the literature. The salient features of these selected OEIC's, all of which operate at a wavelength of $830 \mathrm{~nm}$, are given in Table II. ${ }^{16-20}$

It should be pointed out that if OEIC's and MESFET microwave amplifiers are to be integrated on the same chip it will be necessary to compromise to a single structure for all of the FET's - and therefore of the optical detector - in the interest of ease of fabrication and production yield. The choice that has been made in similar circumstances is to choose a structure with a pinchoff that is midway between the high value that is optimum for high power linear devices and the low value that would be chosen for low power digital circuits. 21 On the other hand, this compromise may not be necessary if the circuit is fabricated on a HEMT or MODFET structure.

\section{CONCLUSIONS}

In this paper we have given some consideration to the general issue of monolithic integration of control circuitry with microwave amplifiers and we have assessed optical detectors whose structures are compatible with GaAs MMIC's. We have seen that detectors of compatible technology, and of adequate speed, for both MESFET and HEMT structures, exist for use in control signal interconnects. We have also found that there are broader issues than detector compatibility that must be addressed before a decision can be made to do large scale integration of microwave, control, and optical components.

\section{REFERENCES}

1. R. C. Goodfellow, B. T. Debney, G. J. Rees, and J. Buus, "Optoelectronic Components for Multigigabit Systems," J. Lightwave Tech., vol. LT-3, pp. 1170-1179, 1985.

2. K. B. Bhasin, G. Anzic, R. R. Kunath, and D. C. Connolly, "Optical Techniques to Feed and Control GaAs MMIC Modules for Phased Array Antenna Applications," AIAA Paper 86-0687-CP, 1987; also published as NASA TM-87218.

3. A. Gupta, G. Kaelin, R. Stein, S. Huston, K. Ip, W. Petersen, M. Miksa, and I. Petroff, "A 20 GHz Phase Shift Transmit Module with 16 dB Gain," IEEE GaAs IC Symposium, pp. 197-200, 1984.

4. T. Gheewala, "GaAs Digital Integrated Circuit Technology," in VLSI Handbook, N. G. Einspruch, ed., (Academic Press, New York, 1985)

5. A. Christou, "GaAs MMIC's: Manufacturing Trends and Issues, "IEEE GaAs IC Symposium, pp. 149-153, 1984.

6. M. Shur, GaAs Devices and Circuits, Plenum Press, New York, 1987.

7. M. Abe, T. Mimura, K. Nishiuchi, A. Shibatomi, and M. Kobayashi, "Recent Advances in UltraHigh-Speed HEMT Technology," IEEE J. Quantum Electron., vol. QE-22, pp. 1870-1880, 1986.

8. A. A. Ketterson, W. T. Masselink, J. S. Gedymin, J. Klem, C. Peng, W. F. Kopp, H. Morkoc, and K. R. Gleason, "Characterization of InGaAs/AlGaAs Pseudomorphic Modulation-Doped Field-Effect Transistors," IEEE Trans. Electron Dev., vol. ED-33, pp. 564-571, 1986.

9. C. W. Slayman and L. Figueroa, "Frequency and Pulse Response of a Novel High Speed Interdigital Surface Photoconductor (IDPC)," IEEE Electron Device Lett., vol. EDL-2, pp. 112-114, 1981.

10. S. J. Wojtczuk, J. M. Ballantyne, S. Wanuga, and Y. K. Chen, "Conparative Study of Easily Integrable Photodetectors," J. Lightwave Tech., vol. LT-5, pp. 1365-1370, 1987.

11. P. C. Claspy and K. B. Bhasin, "Microwave Response of a HEMT Photoconductive Detector," Microwave Optical Tech. Lett., vol. 2, pp. 1-3, 1989.

12. H. Blauvelt, G. Thurmond, J. Parsons, D. Lewis, and H. Yen, "Fabrication and Characterization of GaAs Schottky Barrier Photodetectors for Microwave Fiber Optic Links," Appl. Phys. Lett., vol. 45, pp. 195-197, 1984.

13. M. Ito and O. Wada, "Low Dark Current GaAs Metal-Semiconductor-Metal (MSM) Photodiodes

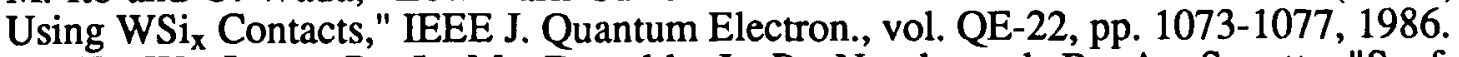

14. D. K. W. Lam, R. I. MacDonald, J. P. Noad, and B. A. Syrett, "Surface-Depleted Photoconductors," IEEE Trans. Electron Dev., vol. ED-34, pp. 1057-1060, 1987. 
15. P. C. Claspy, C. M. Chorey, S. M. Hill, and K. B. Bhasin, "High Frequency GaAlAs Modulator and Photodetector for Phased Array Antenna Applications," SPIE Proceedings, vol. 995, pp. 151$156,1988$.

16. G-K. Chang, H. Schumacher, R. F. Leheny, P. Gerskovich, and S. Nelson, "On-Chip Characterization of Fully Integrated Photoreceivers Using High-Yield Ion-Implanted GaAs IC Technology," 1987 IEEE GaAs IC Symposium, pp. 57-60, 1987.

17. J. D. Crow, C. J. Anderson, S. Bermon, A. Callegari, J. F. Ewen, J. D. Feder, J. H. Greiner, E. P. Harris, P. D. Hoh, H. J. Hovel, J. H. Magerlein, T. E. McKoy, A. T. S. Pomerene, D. L. Rogers, G. J. Scott, M. Thomas, G. W. Mulvey, B. K. Ko, T. Ohashi, M. Scontras, and D. Widiger, "A GaAs MESFET IC for Optical Multiprocessor Networks," IEEE Trans. Electron Dev., vol. 36, pp. 263-268, 1989.

18. M. Makiuchi, H. Hamaguchi, T. Kumai, M. Ito, O. Wada, and T. Sakurai, "A Monolithic FourChannel Photoreceiver Integrated on a GaAs Substrate Using Metal-Semiconductor-Metal Photodiodes and FET's," IEEE Electron Device Lett., vol. EDL-6, pp. 634-636, 1985.

19. C. S. Harder, B. van Zeghbroeck, H. Meier, W. Patrick, and P. Vettiger, "5.2-GHz Bandwidth Monolithic GaAs Optoelectronic Receiver," IEEE Electron Device Lett., vol. 9, pp. 171-173, 1988.

20. G. W. Anderson, N. A. Papanicolaou, D. I Ma, I. A. G. Mack, J. A. Modolo, F. J. Kub, C. W. Young, Jr., P. E. Thompson, and J. B. Boos, "Planar, Linear GaAs Detector-Amplifier Array with an Insulating AlGaAs Spacing Layer Between the Detector and Transistor Layers," IEEE Electron Device Lett., vol. 9, pp. 550-552, 1988.

21. R. L. van Tuyl, V. Kumar, D. C. D'Avanzo, T. W. Taylor, V. E. Peterson, D. P. Hornbuckle, R. A. Fisher, and D. B. Estreich, "A Manufacturing Process for Analog and Digital Gallium Arsenide Integrated Circuits," IEEE Trans. Electron Devices, vol ED-29, pp. 1031-1039, 1982.

Table I. Representative $830 \mathrm{~nm}$ Detectors with MMIC-Compatible Technology

\begin{tabular}{|c|c|c|c|}
\hline Material & Detector type & $3 \mathrm{~dB}$ Frequency & Reference \\
\hline \multicolumn{4}{|c|}{ 1. Ion-implanted MESFET } \\
\hline $\begin{array}{l}\text { SI GaAs, direct on } \\
\text { wafer }\end{array}$ & MSM & $4.5 \mathrm{GHz}$ & Slayman, et. al. $(1981)^{9}$ \\
\hline $\begin{array}{l}\text { SI GaAs, ion } \\
\text { implanted }\end{array}$ & $\begin{array}{l}\text { npn Photodiode } \\
\text { npM Photodiode } \\
\text { Photoconductor }\end{array}$ & $\begin{array}{l}5.1 \\
3.1 \\
0.25\end{array}$ & Wojtczuk, et. al. (1987) ${ }^{10}$ \\
\hline $\begin{array}{l}\text { SI GaAs, ion } \\
\text { implanted }\end{array}$ & $\begin{array}{l}\text { MSM } \\
\text { npn Photodiode } \\
\text { Photoconductor }\end{array}$ & $\begin{aligned} & 4 . \\
> & 7 \\
\approx & 6\end{aligned}$ & Claspy, et. al. (1989) ${ }^{11}$ \\
\hline \multicolumn{4}{|l|}{ 2. Epitaxial MESFET } \\
\hline n-GaAs epi layer & Schottky diode & $>1.3$ & Blauvelt, et. al. $(1984)^{12}$ \\
\hline $\begin{array}{l}\text { GaAs,n-GaAs, } \\
n^{+}-G a A s \text { epi layers }\end{array}$ & MSM & $>1.3$ & Ito, et. al. $(1986)^{13}$ \\
\hline n-GaAs epi layer & Photoconductor & $\approx 20$. & Lam, et. al. $(1987)^{14}$ \\
\hline \multicolumn{4}{|c|}{ 3. AlGaAs/GaAs HEMT (MODFET) } \\
\hline $\begin{array}{l}\mathrm{n}^{+}-\mathrm{AlGaAs} / \mathrm{AlGaAs} \\
\text { GaAs single period }\end{array}$ & Photoconductor & $\approx 2$ & Claspy, et. al. $(1988)^{15}$ \\
\hline
\end{tabular}


TABLE II. Current Status of $830 \mathrm{~nm}$ Optoelectronic Integration

1. Ion implant MESFET Technology

- Bell Communications - Cray OEIC: Chang, et. al. (1987) ${ }^{16}$

- MSM Detector, amplifier

$-3 \mathrm{~dB}$ bandwidth $=1.5 \mathrm{GHz}, 15 \mathrm{~dB}$ amplifier gain

- Fully monolithic chip

- IBM OEIC: Crow, et. al. (1989) ${ }^{17}$

- MSM detector, 4-stage amplifier, clock recovery, serial data recovery

- Operates at $1 \mathrm{Gbit} / \mathrm{sec},-22 \mathrm{dBm}$ optical input for BER $<10^{-9}$

- Fully monolithic chip

- NASA Lewis - Honeywell OEIC: Unpublished

- npn detector, 3-stage amplifier, 1:16 demultiplexer

- Hybrid precursor tested to $300 \mathrm{Mbit} / \mathrm{sec}$, $30 \mathrm{dBm}$ optical input

- Fully monolithic chip to operate at $1 \mathrm{Mbit} / \mathrm{sec}$, being packaged for test

2. Epitaxial MESFET Technology

- Fujitsu Laboratories: Makiuchi, et. al. (1985) ${ }^{18}$

- MSM detector, 2-stage amplifier

- $2 \mathrm{Gbit} / \mathrm{sec} \mathrm{NRZ}$, estimated BER $=10^{-9}$ with $-20 \mathrm{dBm}$ optical input

- Fully monolithic

- IBM Zurich: Harder, et. al. (1988) ${ }^{19}$

- MSM detector, 2-stage amplifier

$-5.2 \mathrm{GHz}$ bandwidth

- Fully monolithic

- Naval Research Laboratory: Anderson, et. al. (1988) ${ }^{20}$

- Photoconductor detector, linear amplifier array (10 elements)

- 650 ps rise time, $1.1 \mathrm{~ns}$ fall time for detector-amplifier combination

- Fully monolithic 


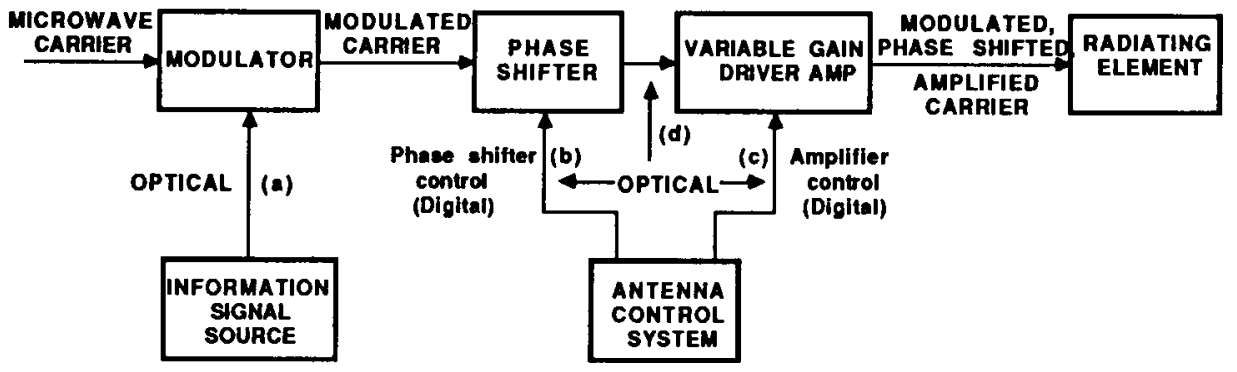

Fig. 1. Schematic of communications system output subsystem showing possible optical interconnects. a, b, and c are wide bandwidth baseband signals, $\mathrm{d}$ is a narrow fractional bandwidth signal based at the system carrier frequency.

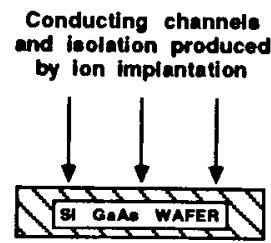

a) ION - IMPLANT MESFET

Isolation by mean etch

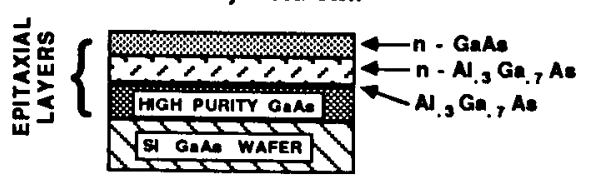

c) HIGH ELECTRON MOBIUTY TRANSISTOR (HEMT)

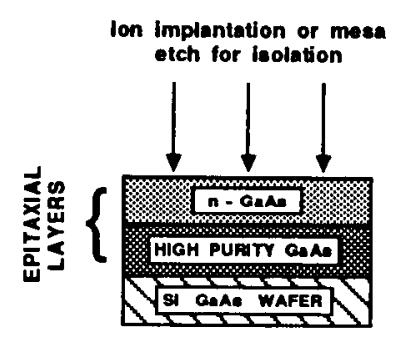

b) EPITAXIAL LAYER MESFET

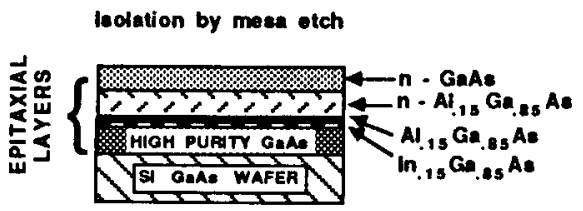

d) PSEUDOMORPHIC HEMT

Fig. 2. Schematic of MMIC material structures and fabrication techniques. 


\section{ORIGINAL PAGE IS \\ OF POOR QUALITY}

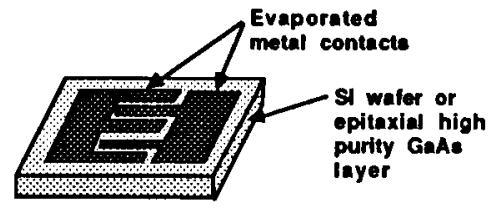

a) Metal - Semiconductor - Metal (MSM)

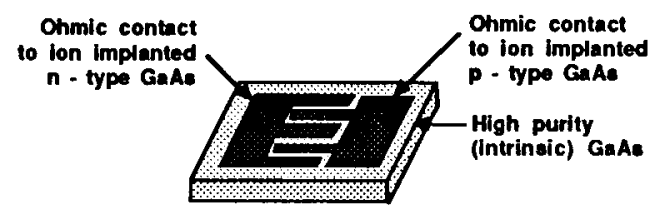

b) $P-1 \cdot N$

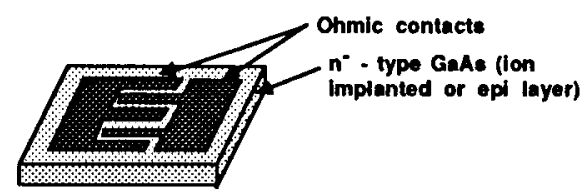

c) Photoconductive

Fig. 3. Schematic of planar detector types.

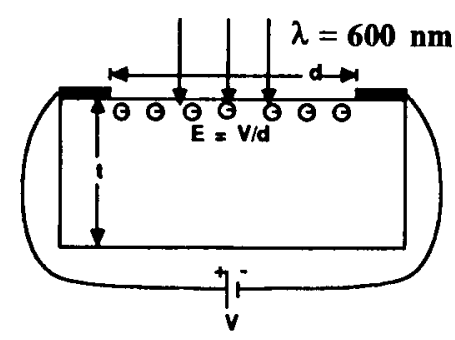

a) Absorption at surface in $\mathrm{GaAs}$ if $\lambda \leq 700 \mathrm{~nm}$ 1 dimensional model OK

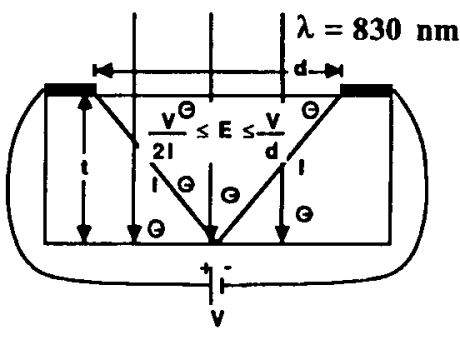

b) Absorption throughout volume in GaAs if $\lambda \geq 700 \mathrm{~nm}$ Must use 2 dimensional model

Fig. 4. Effect of absorption coefficient on detector speed. 


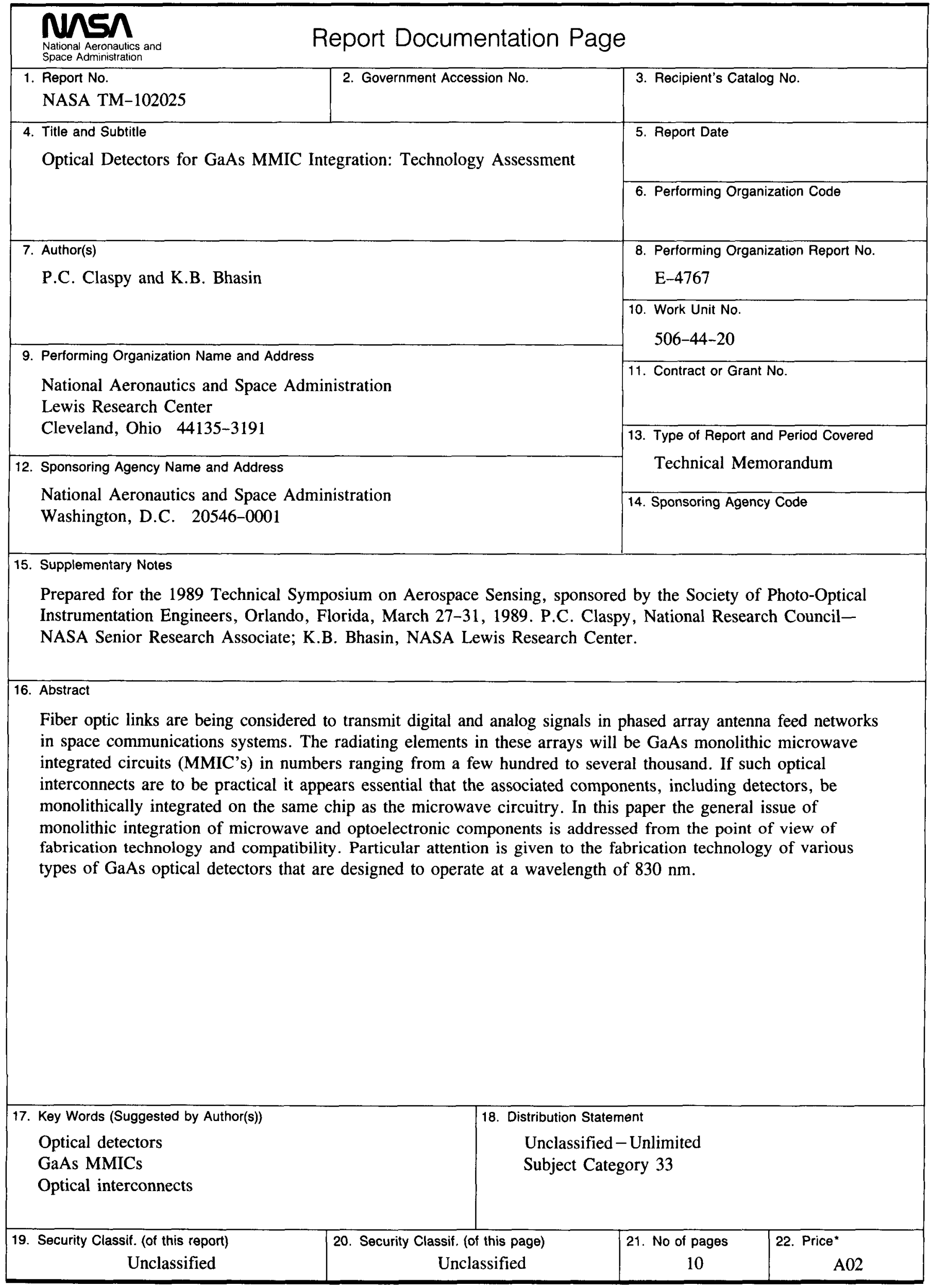

